\title{
RACSAM
}

Rev. R. Acad. Cien. Serie A. Mat.

VOL. 103 (1), 2009, pp. 75-85

Análisis Matemático / Mathematical Analysis

\section{LUR renormings through Deville's Master Lemma}

\section{J. Orihuela and S. Troyanski}

\begin{abstract}
A completely geometrical approach for the construction of locally uniformly rotund norms and the associated networks on a normed space $X$ is presented. A new proof providing a quantitative estimate for a central theorem by M. Raja, A. Moltó and the authors is given with the only external use of Deville-Godefory-Zizler decomposition method.
\end{abstract}

\section{Renormamientos LUR a través del Lema Maestro de Deville}

Resumen. Presentamos una aproximación completamente geométrica para la construcción de normas localmente uniformemente convexas y sus network asociadas en un espacio normado $X$. Se da una nueva demostración, con estimaciones cuantitativas, de un resultado central de M. Raja, A. Moltó y los autores usando únicamente el método de descomposición de Deville-Godefroy-Zizler.

\section{Introduction}

Let $(X,\|\cdot\|)$ be a normed space. The norm $\|\cdot\|$ in $X$ is said to be locally uniformly rotund (LUR for short) if

$$
\left[\lim _{n}\left(2\|x\|^{2}+2\left\|x_{n}\right\|^{2}-\left\|x+x_{n}\right\|^{2}\right)=0\right] \Longrightarrow \lim _{n}\left\|x-x_{n}\right\|=0
$$

for any sequence $\left(x_{n}\right)$ and $x$ in $X$. The construction of this kind of norms in separable Banach spaces lead Kadec to the proof of the existence of homeomorphisms between all separable Banach spaces, [1]. For a non separable Banach space is not always possible to have such an equivalent norm: the space $l^{\infty}$ does not have it, see for instance p. 74 in [2]. When such a norm exists its construction is usually based on a good system of coordinates that we must have on the normed space $X$ from the very beginning, for instance a biorthogonal system,

$$
\left\{\left(x_{i}, f_{i}\right) \in X \times X^{*}: i \in I\right\}
$$

with some additional properties such as being a strong Markushevich basis, [20]. Sometimes there is not such a system and the norm is constructed providing enough convex functions on the given space $X$ adding all of them up with the powerful lemma of Deville, see lemma VII 1.1 in [2]. Deville's lemma has been extensively used by R. Haydon in his seminal papers [6], [7], as well as in [8]. It is based on the construction of an equivalent LUR norm on a weakly compactly generated Banach space by the second named author in [19], where the convex functions are distances to suitable finite dimensional subspaces as well as evaluations on some coordinate functionals in the dual space $X^{*}$; see [20, Theorem 7.3]. We have been able to show the connection between biorthogonal systems and LUR renormings in [17]. Using Deville's lemma we have proved the following:

Presentado por / Submitted by Manuel Valdivia Ureña.

Recibido / Received: 2 de marzo de 2009. Aceptado / Accepted: 4 de marzo de 2009.

Palabras clave / Keywords: LUR renorming, decomposition method, network, completion, slice localization.

Mathematics Subject Classifications: Primary: 46B20; 46B26. Secondary: 46B03, 54C08.

(C) 2009 Real Academia de Ciencias, España. 
Theorem 1 ([17]) Let $X$ be a Banach space and $F \subset X^{*}$ a norming subspace in the dual space $X^{*} . X$ has an equivalent $\sigma(X, F)$-lower semicontinuous and locally uniformly rotund norm if, and only if, there are countably many families of convex and $\sigma(X, F)$-lower semicontinuous functions $\left\{\varphi_{i}^{n}: X \rightarrow \mathbb{R}^{+}\right.$: $\left.i \in I_{n}\right\}_{n=1}^{\infty}$ such that there are open subsets

$$
G_{i}^{n} \subset\left\{\varphi_{i}^{n}>0\right\} \cap\left\{\varphi_{j}^{n}=0: j \neq i, j \in I_{n}\right\}
$$

with $\left\{G_{i}^{n}: i \in I_{n}, n \in \mathbb{N}\right\}$ a basis for the norm topology of $X$.

The method to prove Theorem 1 is mainly based on Stone's theorem about paracompactness of metric spaces, [16]. The $\sigma$-discrete basis for the norm topology of a normed space $X$ can be refined to obtain the basis described in Theorem 1. More recent contributions show an interplay between this method and the one based on Deville's lemma,[7, 11, 12]. It is our intention here to give a straightforward proof of the main renorming construction in $[13,18]$. This result is in the core of the theory, and we shall prove it with a geometrical approach based just on Deville's lemma, without any use of paracompactness at all. Indeed, the Theorem we are going to prove reads as follows:

Theorem $2([18,13])$ Let $X$ be a normed space and $F$ a norming subspace in the dual $X^{*} . X$ admits a $\sigma(X, F)$-lower semicontinuous and equivalent locally uniformly rotund norm if, and only if, there is a sequence $\left(A_{n}\right)$ of subsets of $X$ such that for every $x \in X$ and every $\epsilon>0$ there is a $\sigma(X, F)$-open half space $H$ and a positive integer $p$ with $x \in A_{p} \cap H$ and $\operatorname{diam}\left(A_{p} \cap H\right) \leq \epsilon$.

The known proofs of this result go through a delicate process of convexification of the sets $A_{n}$ needed to construct a countable family of seminorms, and they involve Stone's theorem if additional information on the structure of the sets $A_{n}$ is required, see $[16,18,17]$. We are going to present here a different approach where either Stone's theorem or the convexification process are not needed any more. We shall do it by developing our main result here with the use of Deville's Master Lemma only, indeed we are going to prove the following localization result showing that for any family of slices of a bounded set $A$ of a normed space $X$, we can always construct an equivalent norm such that the LUR condition for a sequence $\left(x_{n}\right)$, and a fixed point $x$ in $A$, implies that the sequence eventually belongs to slices containing the point $x$,too. When the involved slices have small diameter, then the sequence is eventually close to $x$. If the diameter can be made small enough, then the sequence $\left(x_{n}\right)$ converges to $x$ and the norm will be locally uniformly rotund at the point $x$. The precise statement reads as follows:

Theorem 3 (Slice Localization Theorem) Let $X$ be a normed space with a norming subspace $F$ in $X^{*}$. Let $A$ be a bounded subset in $X$ and $\mathcal{H}$ a family of $\sigma(X, F)$-open half spaces such that for every $H \in \mathcal{H}$ the set $A \cap H$ is non empty. Then there is an equivalent $\sigma(X, F)$-lower semicontinuous norm $\|\cdot\|_{\mathcal{H}, A}$ such that for every sequence $\left(x_{n}\right)_{n \in \mathbb{N}}$ in $X$ and $x \in A \cap H$ for some $H \in \mathcal{H}$, if

$$
\lim _{n}\left(2\left\|x_{n}\right\|_{\mathcal{H}, A}^{2}+2\|x\|_{\mathcal{H}, A}^{2}-\left\|x+x_{n}\right\|_{\mathcal{H}, A}^{2}\right)=0,
$$

then there is a sequence of open half spaces $\left\{H_{n} \in \mathcal{H}: n=1,2, \ldots\right\}$ such that

1. There is $n_{0} \in \mathbb{N}$ such that $x, x_{n} \in H_{n}$ for $n \geq n_{0}$ if $x_{n} \in A$.

2. For every $\delta>0$ there is some $n_{\delta}$ such that

$$
x, x_{n} \in{\overline{\left(\operatorname{co}\left(A \cap H_{n}\right)+B(0, \delta)\right)}}^{\sigma(X, F)}
$$

for all $n \geq n_{\delta}$.

We use standard notation in the geometry of Banach spaces and topology that can be found in $[9,4]$ and $[3,10]$. In particular, $B_{X}$ (resp. $S_{X}$ ) is the unit ball (resp. the unit sphere) of a normed space $X$. If $F$ is a subset of $X^{*}$, then $\sigma(X, F)$ denotes the topology of pointwise convergence on $F$. Given $x^{*} \in X^{*}$ and 
$x \in X$, we write $\left\langle x^{*}, x\right\rangle$ and $x^{*}(x)$ to indistinctively denote the evaluation of $x^{*}$ at $x$. If $D$ is a subset of a normed space $X$ we denote by $\operatorname{co}(D)$ the convex hull of $D$. If $x \in X$ and $\delta>0$ we denote by $B(x, \delta)$ the norm open ball centered at $x$ of radius $\delta$. A subspace $F \subset X^{*}$ is said to be a norming subspace in the dual space $X^{*}$ when

$$
\|x\|_{F}:=\sup \left\{\langle x, f\rangle: f \in B_{X^{*}} \cap F\right\}
$$

define an equivalent norm on $X$. When the original norm coincides with $\|\cdot\|_{F}$, the subspace $F$ is called 1-norming.

\section{The tool}

A main result here is Theorem 3 above. It is a refinement of the Connection Lemma we developed in [17]. The difference in the present context is that we do not have any rigidity condition here for the family of slices. In [17] we have slices describing a discrete family of sets. Here we have, instead, an arbitrary family of slices without any additional assumption at all. We need the following definition:

Definition 1 ([17]) Let $X$ be a normed space and $F$ a norming subspace in the dual space $X^{*}$. For a bounded and convex subset $C$ of $X^{* *}$ we define

$$
F-\operatorname{dist}(x, C):=\inf \left\{\sup \left\{\left|<x-c^{* *}, f>\right|: f \in B_{X^{*}} \cap F\right\}: c^{* *} \in C\right\} .
$$

It has been proved in [17] that the $F-\operatorname{dist}(\cdot, C)$ is a convex, $\sigma(X, F)$-lower semicontinuous and 1-Lipschitz map from $X$ to $\mathbb{R}^{+}$. We are going to make extensive use of this kind of functions in our construction of the LUR norm.

Proof of THEOREM 3. We shall consider $\sigma(X, F)$-lower semicontinuous and convex functions $\left(\varphi_{H}\right)$ and $\left(\psi_{H}\right)$ for every $H \in \mathcal{H}$ defined as follows:

$$
\varphi_{H}(x):=F-\operatorname{dist}\left(x,{\overline{H^{c} \cap \operatorname{co}(A)}}^{\sigma\left(X^{* *}, X^{*}\right)}\right)
$$

for every $x \in X$, where we denote by $H^{c}$ the closed half space equal to the complementary of the open half space $H$. Let us choose a point $a_{H} \in H \cap A$ and set $D_{H}=\operatorname{co}(H \cap A)$ for every $H \in \mathcal{H}$, and $D_{H}^{\delta}:=D_{H}+B(0, \delta)$, where $B(0, \delta):=\{x \in X:\|x\|<\delta\}$ for every $\delta>0$ and $H \in \mathcal{H}$. We are going to denote by $p_{H}^{\delta}$ the Minkowski functional of the convex body ${\overline{D_{H}^{\delta}}}^{\sigma(X, F)}-a_{H}$. Then we define the $\sigma(X, F)$-lower semicontinuous norm $p_{H}$ by the formula

$$
p_{H}(x)^{2}=\sum_{n=1}^{\infty} \frac{1}{n^{2} 2^{n}}\left(p_{H}^{1 / n}(x)\right)^{2}
$$

for every $x \in X$. Finally we define the nonnegative, convex, and $\sigma(X, F)$-lower semicontinuous function $\psi_{H}$ as $\psi_{H}(x)^{2}:=p_{H}\left(x-a_{H}\right)^{2}$ for every $x \in X$. We are now in position to apply R. Deville's Master Lemma, see [2, lemma VII.1.1, p. 279], to get an equivalent norm $\|\cdot\|_{\mathcal{H}, A}$ on $X$ such that the condition

$$
\lim _{n}\left(2\left\|x_{n}\right\|_{\mathcal{H}, A}^{2}+2\|x\|_{\mathcal{H}, A}^{2}-\left\|x_{n}+x\right\|_{\mathcal{H}, A}^{2}\right)=0
$$

for a sequence $\left\{x_{n}: n \in \mathbb{N}\right\}$ and $x$ in $X$ implies that there exists a sequence of indexes $\left(H_{n}\right)$ in $\mathcal{H}$ such that

1. $\lim _{n} \varphi_{H_{n}}(x)=\lim _{n} \varphi_{H_{n}}\left(x_{n}\right)=\lim _{n} \varphi_{H_{n}}\left(\left(x+x_{n}\right) / 2\right)=\sup \left\{\varphi_{H}(x): H \in \mathcal{H}\right\}$ and

2. $\lim _{n}\left[(1 / 2) \psi_{H_{n}}^{2}\left(x_{n}\right)+(1 / 2) \psi_{H_{n}}^{2}(x)-\psi_{H_{n}}^{2}\left(\left(x_{n}+x\right) / 2\right)\right]=0$ 
If the given point $x$ belongs to one of the open half spaces $H_{0} \in \mathcal{H}_{\epsilon}$ then we have that $\varphi_{H_{0}}(x)>0$ and so we have that:

$$
\sup \left\{\varphi_{H}(x): H \in \mathcal{H}_{\epsilon}\right\} \geq \varphi_{H_{0}}(x)>0,
$$

condition 1 provide us with an integer $n_{0}$ such that

$$
\varphi_{H_{n}}(x)>0, \quad \varphi_{H_{n}}\left(x_{n}\right)>0, \quad \varphi_{H_{n}}\left(\left(x+x_{n}\right) / 2\right)>0
$$

whenever $n \geq n_{0}$, from where our conclusion 1 in the Theorem follows. Moreover, condition 2 above and standard convexity arguments imply now that for every positive integer $q$ we have that

$$
\lim _{n}\left[(1 / 2)\left(p_{H_{n}}^{1 / q}\left(x_{n}-a_{H_{n}}\right)\right)^{2}+(1 / 2)\left(p_{H_{n}}^{1 / q}\left(x-a_{H_{n}}\right)\right)^{2}-\left(p_{H_{n}}^{1 / q}\left(\left(x_{n}+x\right) / 2-a_{H_{n}}\right)\right)^{2}\right]=0,
$$

consequently,

$$
\lim _{n}\left[p_{H_{n}}^{1 / q}\left(x_{n}-a_{H_{n}}\right)-p_{H_{n}}^{1 / q}\left(x-a_{H_{n}}\right)\right]=0, \quad \forall q \in \mathbb{N}-
$$

If we fix a positive number $\delta$, an open half space $H \in \mathcal{H}$ and $y \in A \cap H$ we have that

$$
y-a_{H}+\left(y-a_{H}\right) \delta\left\|y-a_{H}\right\|^{-1} \in B(0, \delta)+\left(y-a_{H}\right) \subset D_{H}^{\delta}-a_{H},
$$

thus

$$
\left[(1+\delta)\left\|y-a_{H}\right\|^{-1}\right]\left(y-a_{H}\right) \in\left(D_{H}^{\delta}-a_{H}\right)
$$

and therefore

$$
p_{H}^{\delta}\left(y-a_{H}\right)<\left[\left(1+\delta\left\|y-a_{H}\right\|^{-1}\right]^{-1}\right.
$$

since $D_{H}^{\delta}-a_{H}$ is a norm open set.

Let us choose now the integer $q$ such that $1 / q<\delta$, and take an integer $n \geq n_{0}$. We know that $x \in A \cap H_{n}$ since $\varphi_{H_{n}}(x)>0$ and the given point $x$ belongs to $A$. Therefore

$$
p_{H_{n}}^{1 / q}\left(x-a_{H_{n}}\right)<\left[\left(1+(1 / q)\left\|x-a_{H_{n}}\right\|^{-1}\right]^{-1},\right.
$$

and we can find a number $0<\xi<1$ such that

$$
p_{H_{n}}^{1 / q}\left(x-a_{H_{n}}\right)<1-\xi,
$$

for all $n \geq n_{0}$, by the boundness of $A$. If we now take the integer $n$ big enough to have

$$
p_{H_{n}}^{1 / q}\left(x_{n}-a_{H_{n}}\right)<1-\xi,
$$

we arrive to the fact that $x_{n}-a_{H_{n}} \in D_{H_{n}}^{\delta}-a_{H_{n}}$, and indeed $x_{n} \in{\overline{\left(\operatorname{co}\left(A \cap H_{n}\right)+B(0, \delta)\right)}}^{\sigma(X, F)}$, so the proof is over.

Thus, given any family of slices on a given set $A$ of a normed space, we have seen how it is always possible to construct equivalent norms such that the LUR condition on a given sequence $\left(x_{n}\right)$ and a fixed point $x$ implies that the sequence eventually belongs to halfspaces of the given family containing the point $x$ too.

\section{LUR renormings}

We can prove now a quantitative version for the main results in [13] and [18]. It corresponds with the renorming implication of Theorem 2, where the hypothesis provide the conclusion for every $\epsilon>0$ and every $x \in X$. 
Theorem 4 Let $X$ be a normed space and $F \subset X^{*}$ be a 1-norming subspace for $X$. Given $\epsilon$ we assume that there are subsets $A_{n}$ such that for every $x \in \cup_{n=1}^{\infty} A_{n}$ we can find $p \in \mathbb{N}$ and a $\sigma(X, F)$-open half space $H$ such that $x \in A_{p} \cap H$ and $\operatorname{diam}\left(A_{p} \cap H\right) \leq \epsilon$. Then $X$ admits an equivalent $\sigma(X, F)$-lower semicontinuous norm $\||\cdot|\|$ such that the condition

$$
\lim _{n}\left(2\left\|\left|x_{n}\right|\right\|^{2}+2 \mid\|x\|^{2}-\left\|x+x_{n}\right\|^{2}\right)=0
$$

implies that for every $\delta>0$ there is some integer $n_{\delta}$ such that for all $n \geq n_{\delta}$ we have $\left\|x_{n}-x\right\|<\epsilon+\delta$ whenever $x \in \cup_{n=1}^{\infty} A_{n}$.

PRoof. Let us consider the family $\mathcal{H}_{n}$ of all $\sigma(X, F)$-open half spaces such that $A_{n} \cap H \neq \emptyset$ and $\operatorname{diam}\left(A_{n} \cap H\right) \leq \epsilon$. If there is not such slice for some set $A_{n}$ we do not consider it at all. If we apply the former Theorem for the family $\mathcal{H}_{n}$ and the set $A_{n}$ we get an equivalent norm $\|\cdot\|_{n}$ that verifies conditions 1 and 2 of Theorem 3 for any sequence $\left(x_{m}\right)$ and $x$ such that

$$
\lim _{m}\left(2\left\|x_{m}\right\|_{n}^{2}+2\|x\|_{n}^{2}-\left\|x+x_{m}\right\|_{n}^{2}\right)=0
$$

Let us take $c_{n}$ such that $\|\cdot\|_{n} \leq c_{n}\|\cdot\|$. If we set

$$
\|x \mid\|^{2}:=\sum_{n=1}^{\infty} \frac{1}{c_{n} 2^{n}}\|x\|_{n}^{2}
$$

for every $x \in X$, we obtain the renorming we are looking for. Indeed, if

$$
\lim _{n}\left(2 \| | | x _ { n } | | ^ { 2 } + 2 \| \left\|x\left|\left\|^{2}-\right\|\left\|\left|x+x_{n}\right| \mid\right\|^{2}\right)=0\right.\right.
$$

by standard convex arguments we know that

$$
\lim _{n}\left(2\left|\left\|x_{n}\right\|_{p}^{2}+2\right|\left\|x\left|\left\|_{p}^{2}-\right\|\right| x+x_{n}\right\|_{p}^{2}\right)=0
$$

for every positive integer $p$. If $x \in A_{q}$ and there is a $\sigma(X, F)$-open half space $H$ such that $x \in A_{q} \cap H$ and $\operatorname{diam}\left(A_{q} \cap H\right) \leq \epsilon$, we have that $H \in \mathcal{H}_{q}$ and $\operatorname{diam}\left(\operatorname{co}\left(A_{p} \cap H\right)\right) \leq \epsilon$, too. Moreover, the condition 2 of Theorem 3 tell us that there is a sequence of half spaces $H_{n} \in \mathcal{H}_{q}$ such that for every $\delta>0$ there is some $n_{\delta}$ with

$$
x, x_{n} \in{\overline{\left(\operatorname{co}\left(A_{q} \cap H_{n}\right)+B(0, \delta)\right)}}^{\sigma(X, F)}
$$

for all $n \geq n_{\delta}$. Since $F$ is 1 -norming the original norm is $\sigma(X, F)$-lower semicontinuous and we have $\left\|x-x_{n}\right\| \leq \epsilon+\delta$ for every $n \geq n_{\delta}$, as we wanted to prove.

Corollary 1 Let $X$ be a Banach space and $F \subset X^{*}$ a norming subspace for $X$. Let us assume that $Z \subset X$ is a subspace of $X$ with a sequence of subsets $\left(A_{n}\right) \subset Z$ such that for every $\epsilon>0$ and $z \in Z$ there is some $p \in \mathbb{N}$ together with a $\sigma(X, F)$-open half space $H$ such that $z \in H \cap A_{p}$ and $\operatorname{diam}\left(A_{p} \cap H\right) \leq \epsilon$. Then the whole space $X$ admits an equivalent $\sigma(X, F)$-lower semicontinuous norm $\|\cdot|\||$ such all points in the subspace $Z$ are $\mathbf{L U R}$ points for the new norm in the whole of $X$, i.e. for every point $z \in Z$ and every sequence $\left(x_{n}\right)$ in $X$ such that

$$
\lim _{n}\left(2\|\| x_{n}\left|\left\|^{2}+2\right\|\|z\|^{2}-\left\||| z+x_{n}\right\|^{2}\right)=0\right.
$$

we will have that $\lim _{n} x_{n}=z$ in norm. 
PROOF. Without loss of generality we may and do assume that the original norm is $\|\cdot\|_{F}$; i.e. $F$ is a 1 norming subspace. If we perform the construction in Theorem 4 for a fixed $\epsilon>0$ we obtain the equivalent $\sigma(X, F)$-lower semicontinuous norm $\|\cdot\|_{\epsilon}$. Let us take $d_{n}$ such that $\|\cdot\|_{1 / n} \leq d_{n}\|\cdot\|_{1 / n}$. If we set

$$
\|x\|^{2}:=\sum_{n=1}^{\infty} \frac{1}{d_{n} 2^{n}}\|x\|_{1 / n}^{2}
$$

for every $x \in X$, we obtain the renorming we are looking for. Indeed, as above, if

$$
\lim _{n}\left(2\|\| x_{n} \mid\left\|^{2}+2\right\|\|z\|^{2}-\|\| z+x_{n} \|^{2}\right)=0
$$

by standard convex arguments we know that

$$
\lim _{n}\left(2\left|\left\|x_{n}\right\|_{1 / p}^{2}+2\|\| z\left\|_{1 / p}^{2}-\right\|\right| \mid z+x_{n} \|_{1 / p}^{2}\right)=0
$$

for every positive integer $p$, and Theorem 4 says that $\left\|x_{n}-z\right\| \|<2 / p$ for $n \geq n_{1 / p}$ whenever $z \in Z$.

Remark 1 The corollary provide us with a geometrical proof of the renorming implication in Theorem 2 based on the Deville-Godefroy-Zizler decomposition method only.

Corollary 2 Let $X$ and $Y$ be normed spaces with 1-norming subspaces $F \subset X^{*}, G \subset Y^{*}$ and

$$
T: X \longrightarrow Y
$$

a bounded linear map, continuous for the $\sigma(X, F)$ and $\sigma(Y, G)$ topologies. Given $\epsilon>0$ we assume there are subsets $A_{n} \subset Y$ such that for every $y \in \cup_{n=1}^{\infty} A_{n}$ we can find $p \in \mathbb{N}$ and a $\sigma(Y, G)$-open half space $L$ such that $y \in A_{p} \cap L$ and $\operatorname{diam}\left(A_{p} \cap L\right) \leq \epsilon$. Then $X$ admits an equivalent $\sigma(X, F)$-lower semicontinuous norm $\|\cdot \mid\| \|_{T}$ such that the condition

$$
\lim _{n}\left(2\left|\left\|x_{n}\right\|_{T}^{2}+2\right|\|x\|_{T}^{2}-\left\|\mid x+x_{n}\right\|_{T}^{2}\right)=0
$$

implies that for every $\delta>0$ there is some integer $n_{\delta}$ such that for all $n \geq n_{\delta}$ we have

$$
\left\|T\left(x_{n}\right)-T(x)\right\| \mid<\epsilon+\delta\|T\|
$$

whenever $T(x) \in \cup_{n=1}^{\infty} A_{n}$. In particular, when $Y$ admits an equivalent $\sigma(Y, G)$-lower semicontinuous and $\mathbf{L U R}$ norm we will have that the condition

$$
\lim _{n}\left(2\left\|\mid x_{n}\right\|\left\|_{T}^{2}+2\right\|\|x\|_{T}^{2}-\left\|x+x_{n}\right\|_{T}^{2}\right)=0
$$

implies that $\lim _{n} T\left(x_{n}\right)=T(x)$ in the norm of $Y$.

ProOF. Let us fix the integer $n$ and apply Theorem 3 to the set $T^{-1}\left(A_{n}\right)$ together with the family $\mathcal{H}_{n}$ of $\sigma(X, F)$-open half spaces given by $T^{-1}(L)$ for every $L$, a $\sigma(Y, G)$-open half space, such that $A_{n} \cap L \neq \emptyset$ and $\operatorname{diam}\left(A_{n} \cap L\right) \leq \epsilon$. We will get an equivalent norm $\|\cdot\|_{n}$ on $X$ such that, the condition

$$
\lim _{m}\left(2\left\||| x_{m}\right\|_{n}^{2}+2\|\| x\left\|_{n}^{2}-\right\| \mid x+x_{m} \|_{n}^{2}\right)=0
$$

implies that $x_{m}$ and $x$ are in the sets

$$
{\overline{\operatorname{co}\left(T^{-1}\left(A_{n}\right) \cap T^{-1}\left(L_{m}\right)\right)+B(0, \delta)}}_{\sigma(Y, G)}
$$

for $m \geq m_{\delta}$, where $\operatorname{diam}\left(A_{n} \cap L_{m}\right) \leq \epsilon$, and therefore $\left\|T\left(x_{m}\right)-T(x)\right\| \leq \epsilon+\delta\|T\|$ for all $m \geq m_{\delta}$ whenever $T(x) \in A_{n} \cap L$ with some $\sigma(Y, G)$-open half space $L$ and $\operatorname{diam}\left(A_{n} \cap L\right) \leq \epsilon$. Adding all this 
norms we get the equivalent norm $\|\cdot\|_{T}$ we are looking for. Indeed, let us take $h_{n}$ such that $\|\cdot\|_{n} \leq h_{n}\|\cdot\|$; if we set

$$
\|x\|^{2}:=\sum_{n=1}^{\infty} \frac{1}{h_{n} 2^{n}}\|x\|_{n}^{2}
$$

for every $x \in X$, we obtain the renorming we are looking for. The proof follows the same arguments as above. When Y admits the LUR norm we have the former conditions for all $\epsilon>0$ by Theorem 2, and the conclusion then follows.

\section{The network construction}

Our approach for LUR renormings is also based on the topological concept of network. A family of subsets $\mathcal{N}$ in a topological space $(T, \mathcal{T})$ is a network for the topology $\mathcal{T}$ if for every open set $W \in \mathcal{T}$, and every $x \in W$, there is some $N \in \mathcal{N}$ such that $x \in N \subset W$.

Let us recall the following definitions and results:

Definition 2 Let $X$ be a normed space and $F$ a norming subspace in the dual $X^{*}$. A family $\mathcal{B}:=$ $\left\{B_{i}: i \in I\right\}$ of subsets on $X$ is called $\sigma(X, F)$-slicely isolated (or $\sigma(X, F)$-slicely relatively discrete) if it is a disjoint family of sets such that for every

$$
x \in \bigcup\left\{B_{i}: i \in I\right\}
$$

there exist a $\sigma(X, F)$-open half space $H$ and $i_{0} \in I$ such that

$$
H \bigcap \bigcup\left\{B_{i}: i \in I, i \neq i_{0}\right\}=\emptyset \quad \text { and } \quad x \in B_{i_{0}} \cap H
$$

A main result, obtained by using the approach of [16], is the following one; it is equivalent to Theorem 2 if we have in mind Stone's theorem on the paracompactness of a metric space, see [16, chapter III].

Theorem 5 ([16, chapter III, Theorem 3.1, pag 49]) Let $X$ be a normed space and $F$ a norming subspace in the dual $X^{*}$. The space $X$ admits an equivalent $\sigma(X, F)$-lower semicontinuous and locally uniformly rotund norm if, and only if, the norm topology has a network $\mathcal{N}$ that can be written as $\mathcal{N}=\cup_{n=1}^{\infty} \mathcal{N}_{n}$ where each of the families $\mathcal{N}_{n}$ is $\sigma(X, F)$ - slicely isolated.

In the monograph [16] the network point of view for locally uniformly rotund renormings is the central one. The approach to construct networks make extensive use of Stone's theorem on the paracompactness of metric spaces. We shall construct in this section the network that characterize the property of being locally uniformly rotund renormable, but our approach will be completely geometrical as the one presented in [14] for the weak topology, see lemma 3.19 in [16] too. We have presented the next result in [17] but using Stone's theorem in the construction.

Theorem 6 Let $X$ be a normed space with a $\sigma(X, F)$-lower semicontinuous and locally uniformly rotund norm for some subspace $F \subset X^{*}$. Then the norm topology admits a network $\mathcal{N}$ such that $\mathcal{N}=\cup_{n=1}^{\infty} \mathcal{N}_{n}$ where the families $\mathcal{N}_{n}$ are norm discrete, $\sigma(X, F)$-slicely isolated, and consisting of sets which are the difference of convex and $\sigma(X, F)$-closed subsets of $X$ for every $n \in \mathbb{N}$.

PROOF. In a LUR norm all points in the unit sphere are denting points, then for $\epsilon>0$ fixed we will have a family of $\sigma(X, F)$-open half spaces $\mathcal{H}_{\epsilon}$, covering the unit sphere $S_{X}$ of our $\sigma(X, F)$-lower semicontinuous and LUR norm, and such that $\|\cdot\|-\operatorname{diam}\left(H \cap B_{X}\right)<\epsilon$ for all $H \in \mathcal{H}_{\epsilon}$. Let us choose a well order relation for the elements in $\mathcal{H}_{\epsilon}$ and let us write

$$
\mathcal{H}_{\epsilon}=\left\{H_{\gamma}: \gamma<\Gamma\right\}
$$


where we denote $H_{\gamma}=\left\{x \in X: f_{\gamma}(x)>\lambda_{\gamma}\right\}, f_{\gamma} \in B_{X^{*}} \cap F$.

We set

$$
M_{\gamma}:=H_{\gamma} \cap B_{X} \backslash\left(\bigcup\left\{H_{\beta} \cap B_{X}: \beta<\gamma\right\}\right)
$$

for every $\gamma<\Gamma$. Let us define the sets $M_{\gamma}^{n}:=\left\{x \in M_{\gamma}: f_{\gamma}(x) \geq \lambda_{\gamma}+1 / n\right\}$. It follows that, when $x \in M_{\gamma}^{n}$ and $y \in M_{\beta}^{n}$ for $\gamma \neq \beta$ then we have either

$$
f_{\gamma}(x)-f_{\gamma}(y) \geq 1 / n \quad(\text { when } \gamma<\beta),
$$

or

$$
f_{\beta}(y)-f_{\beta}(x) \geq 1 / n \quad(\text { when } \beta<\gamma),
$$

but in any case

$$
\|x-y\| \geq 1 / n
$$

since the linear functionals $f_{\gamma}, f_{\beta}$ are assumed to be in $B_{X^{*}} \cap F$. If we fix $x \in S_{X}$ the LUR condition of the norm gives a slice

$$
G=\left\{y \in B_{X}: g(y)>\mu\right\}
$$

with $g(x)>\mu, g \in B_{X^{*}} \cap F$ and $\|\cdot\|-\operatorname{diam}(G)<1 / n$, thus $G$ meets at most one member of the family of sets $\left\{M_{\gamma}^{n}: \gamma<\Gamma\right\}$ by (3).

These families of closed and convex subsets of $X$ cover the unit sphere $S_{X}$ and they suffice to describe the network there. Nevertheless, to go over the whole space $X$ we need to make the difference of closets convex sets. Indeed, take $x \in X \backslash\{0\}$, and $y:=x /\|x\|$. If we take $\gamma_{0}<\Gamma$ so that $y \in M_{\gamma_{0}}$ and $n$ big enough to have $f_{\gamma_{0}}(y)>\lambda_{\gamma_{0}}+1 / n$, we will have a rational number $0<\mu_{x}<1$, close enough to one, such that $f_{\gamma_{0}}\left(\mu_{x} y\right)>\lambda_{\gamma_{0}}+1 / n$. The LUR condition of the norm tell us that there is $\delta_{x}>0$ such that $\|(y+z) / 2\|>1-\delta_{x}$ implies that $\|y-z\|<1 / n$ whenever the condition $\|z\| \leq 1$ holds.

Let us take a rational number $\rho$ such that

$$
\rho>\|x\|>\rho\left(1-\delta_{x}\right) \quad \text { and } \quad \rho \mu_{x}<\|x\| .
$$

Then $x \in \rho M_{\gamma_{0}}^{n}$ and $\|\cdot\|-\operatorname{diam}\left(\rho M_{\gamma_{0}}^{n}\right)<\rho \epsilon$. Moreover, if we choose $g_{x} \in B_{X^{*}} \cap F$ such that $g_{x}(x)>\rho\left(1-\delta_{x}\right)$ then, for any $z \in \cup\left\{\rho M_{\gamma}^{n}: \gamma<\Gamma\right\}$ with $g_{x}(z)>\rho\left(1-\delta_{x}\right)$, we will have

$$
g_{x}(z / \rho)>1-\delta_{x} \quad \text { and } \quad g_{x}(y)>\frac{\rho\left(1-\delta_{x}\right)}{\|x\|}>1-\delta_{x},
$$

thus $\left\|\frac{y+z / \rho}{2}\right\|>1-\delta_{x}$, and we will have $\|y-z / \rho\|<1 / n$, from where it follows that $\gamma=\gamma_{0}$. Therefore, if we consider sets $M_{\gamma}^{n, p}:=\left\{x \in M_{\gamma}^{n} \cap S_{X}: \delta_{x}>1 / p\right\}$, and we take the family

$$
\left\{\rho M_{\gamma}^{n, p} \backslash \rho(1-1 / p) B_{X}: \gamma<\Gamma\right\}
$$

for rational numbers $\rho$ and integers $p, n$ fixed, we form an slicely isolated family of sets. All together, with the same construction done for every $\epsilon>0$ we obtain a family

$$
\bigcup\left\{\left\{\rho M_{\gamma}^{n, p}(\epsilon) \backslash \rho(1-1 / p) B_{X}: \gamma<\Gamma\right\}: \rho \in \mathbb{Q}, n, p \in \mathbb{N}, \epsilon>0\right\}
$$

which is a network for the norm topology. Taking $\epsilon=1 / r, r=1,2, \ldots$ we get the network for the norm we are looking for.

Remark 2 Let us observe that we have completed a geometrical proof of Theorem 2. Indeed, Theorem 6 provides us with the $\sigma(X, F)$-slicely isolated network $\mathcal{N}=\cup_{n=1}^{\infty} \mathcal{N}_{n}$ for the norm topology. Setting $A_{q}:=$ $\cup\left\{N: N \in \mathcal{N}_{q}\right\}$ for $q \in \mathbb{N}$ and given $x \in X$ and $\epsilon>0$, if we take $p$ and $M \in \mathcal{N}_{p}$ with $x \in M \subset$ $B(x, \epsilon / 2)$, then by the slicely isolatedness property of the family $\mathcal{N}_{p}$ there is a $\sigma(X, F)$-open half space $H$ with $x \in H \cap A_{p} \subset M$. Thus $\|\cdot\|-\operatorname{diam}\left(H \cap A_{p}\right) \leq\|\cdot\|-\operatorname{diam}(M) \leq \epsilon$. The reverse implication follows from corollary 1 . 
Let us remark that the space $l_{\infty}$ is a complemented subspace of a Banach space $X$ with a Markushevich basis $\left\{\left(x_{i}, f_{i}\right) \in X \times X^{*}: i \in I\right\}$ by a result of Plichko, [4, Theorem 6.45], and that $l_{\infty}$ does not admit equivalent LUR norm, [2, Theorem II.7.10]. It follows that the linear span

$$
E:=\operatorname{span}\left\{x_{i}: i \in I\right\}
$$

give us an example of a normed space with an equivalent LUR norm, [5, Theorem 3.48] or [15, example 2], since the proof is valid for a normed space, but such that its completion $X$ doest not have it.

The network provides us with criteria to see when the closure of a LUR renormable space could be LUR renormable too. For instance, we can prove the following:

Theorem 7 Let $E$ be a normed space with a norming subspace $F \subset E^{*}$ and $X$ its completion. There is a network $\mathcal{N}=\cup \mathcal{N}_{n}$ of the norm topology on $E$ where each of the families $\mathcal{N}_{n}$ is $\sigma(E, F)$-slicely isolated and such that the family of sets:

$$
\mathcal{B}:=\left\{N+\epsilon B_{X}: N \in \mathcal{N}, \epsilon>0\right\}
$$

is a basis for the norm topology of $X$ if, and only if, the completion $X$ admits an equivalent $\sigma(X, F)$-lower semicontinuous and LUR equivalent norm.

In the proof we are going to use the following result that we have obtained in [17].

Proposition 1 Let $X$ be a normed space with a norming subspace $F \subset X^{*}$ and $\|\cdot\|_{F}$ the equivalent norm associated with it. Given a $\sigma(X, F)$-slicely isolated family $\mathcal{A}:=\left\{A_{i}: i \in I\right\}$ there exist decompositions with increasing sequences of subsets $\left(A_{i}^{n}\right)_{n}, A_{i}=\cup_{n=1}^{\infty} A_{i}^{n}$ for every $i \in I$, such that the families

$$
\left\{A_{i}^{n}+B_{\|\cdot\|_{F}}(0,1 / 4 n): i \in I\right\}
$$

are $\sigma(X, F)$-slicely isolated and norm discrete for every $n \in \mathbb{N}$.

ProOF OF THEOREM 7. If the normed space $X$ admits an equivalent $\sigma(X, F)$-lower semicontinuous and LUR norm, we have proved in [17] that it has a basis of the norm topology $\mathcal{B}=\cup \mathcal{B}_{n}$ such that every one of the families of open sets $\mathcal{B}_{n}$ is $\sigma(X, F)$-slicely isolated and norm discrete. It now follows that $\mathcal{N}_{n}:=\mathcal{B}_{n} \cap F$ are families of non- void subsets in $E$ since $E$ is dense in $X$, and they are $\sigma(E, F)$-slicely isolated and norm discrete with $\mathcal{N}:=\cup_{n=1}^{\infty} \mathcal{N}_{n}$ a basis of the norm topology of $E$. It is clear that the family of sets

$$
\mathcal{B}:=\left\{N+\epsilon B_{X}: N \in \mathcal{N}, \epsilon>0\right\}
$$

is a basis of the norm topology of $X$. Indeed, since every set $B \in \mathcal{B}$ is open and $E$ is dense we have $B \subset \overline{B \cap E}$. This fact together with the regularity of the norm topology complete the proof for this implication.

Let us prove now the converse result. Without loss of generality we can assume that the given norm in $X$ coincides with $\|\cdot\|_{F}$. Let us fix a $\sigma$-slicely isolated (for $\sigma(E, F)$ ) network $\mathcal{N}$ of the norm topology in $E$ such that the family of sets

$$
\mathcal{B}:=\left\{N+\epsilon B_{X}: N \in \mathcal{N}, \epsilon>0\right\}
$$

is a basis of the norm topology of $X$. Let us write $\mathcal{N}=\cup \mathcal{N}_{n}$ where each of the families $\mathcal{N}_{n}$ is a $\sigma(E, F)$ slicely isolated family of sets in $E$, thus $\sigma(X, F)$-slicely isolated in $X$, too. We apply the Proposition 1 and we can write:

$$
\mathcal{N}_{n}:=\left\{N_{j}^{n}: j \in I_{n}\right\},
$$

$N_{i}^{n}=\bigcup_{m=1}^{\infty} N_{i}^{n}(m)$, where $N_{i}^{n}(1) \subset N_{i}^{n}(2) \subset \cdots \subset N_{i}^{n}(m) \subset \cdots$ and the families

$$
\left.\left\{N_{i}^{n}(m)+(1 / 4 m) B_{X}\right): i \in I_{n}\right\},
$$


for every fixed integer $m$, are $\sigma(X, F)$-slicely isolated and norm discrete. Moreover, the families

$$
\bigcup_{n, m \in \mathbb{N}}\left\{\overline{N_{i}^{n}(m)}: i \in I_{n}\right\}
$$

form a $\sigma(X, F)$-slicely isolated network of the norm topology on the whole space $X$ as we are going to see now. Let us take $x \in X$ and $\epsilon>0$. Then there is some pair of positive integers $p, q$ such that $x \in \bar{N}_{i}^{p}(q) \subset B(x, \epsilon)$ for some $i \in I_{p}$. Indeed, if not we will have some point $x_{p, q} \in \overline{N_{i}^{p}(q)} \cap(X \backslash B(x, \epsilon))$ whenever

$$
x \in N_{i}^{p}(q)+\delta B_{X} \subset B(x, \epsilon)
$$

for some $p, q \in \mathbb{N}$, some $i \in I_{p}$ and some $\delta>0$. Let us begin with the first integers $p_{1}$ such that

$$
x \in N_{i}+\delta_{1} B_{X} \subset B(x, \epsilon)
$$

for some $i \in I_{p_{1}}$ and some $\delta_{1}>0$. Thus we can select the first integer $q_{1}$ such that

$$
x \in N_{i}^{p_{1}}\left(q_{1}\right)+\delta_{1} B_{X} \subset B(x, \epsilon)
$$

and take $x_{1} \in \overline{N_{i}^{p_{1}}\left(q_{1}\right)} \cap(X \backslash B(x, \epsilon))$ by our assumption. Taking $0<\delta_{2}$ small enough we will have $B\left(x, \delta_{2}\right) \subset B(x, \epsilon)$ too. Let us take again first integers $p_{2}$ such that

$$
x \in N_{i}+\delta_{3} B_{X} \subset B\left(x, \delta_{2}\right),
$$

for some $i \in I_{p_{2}}$ and some $\delta_{3}>0$ together with the first integer $q_{2}$ such that

$$
x \in N_{i}^{p_{2}}\left(q_{2}\right)+\delta_{3} B_{X} \subset B\left(x, \delta_{2}\right),
$$

then we can take again a point $x_{2} \in \overline{N_{i}^{p_{1}}\left(q_{1}\right)} \cap(X \backslash B(x, \epsilon))$ together with $0<\delta_{4}<\delta_{2} / 2$. If we continue in this way, by induction we obtain a sequence $\left(x_{n}\right)$ in the closed set $X \backslash B(x, \epsilon)$ with a decreasing sequence $\left(\delta_{2 n}\right) \downarrow 0$ such that $x_{n} \in B\left(x, \delta_{2 n}\right)$, a contradiction, and the proof is over.

Acknowledgement. Supported in part by Project MTM2008-05396/MTM Fondos FEDER (J. Orihuela and S. Troyanski). Supported in part by Fundación Séneca 08848/PI/08 CARM (J. Orihuela and S. Troyanski) Supported in part by Institute of Mathematics and Informatics of the Bulgarian Academy of Sciences, and grant of the Bulgarian National Fund for Scientific Research contract DO $02-360 / 2008$ (S. Troyanski).

\section{References}

[1] Bessaga, C. And Pelczynski, A., (1975). Selected Topics in Infinite-dimensional Topology Monografie Matematyczne, vol 58, PWN-Polish Scientific Publishers.

[2] Deville, R., Godefroy, G. And Zizler, V., (1993). Smoothness and renormings in Banach spaces, Pitman Monographs and Surveys in Pure and Applied Mathematics, vol. 64, New York.

[3] Engelking, R., (1977). General topology, PWN-Polish Scientific Publishers, Warsaw, Translated from the Polish by the author, Monografie Matematyczne, Tom 60. [Mathematical Monographs, Vol. 60].

[4] Fabian, M., Habala, P., Hájek, P., Montesinos, V., Pelant, J. and Zizler, V., (2001). Functional Analysis and Infinite Dimensional Geometry, CMS Books in Mathematicas, Springer Verlag, New York

[5] Hájek, P., Montesinos, V., Vanderwerff, J. And Zizler, V., (2008). Biorthogonal Systems in Banach spaces, CMS Books in Mathematics, Springer Verlag, New York.

[6] Haydon, R., (1999). Trees in renorming theory, Proc. London Math. Soc., 78, (3), 541-584. 
[7] Haydon, R., (2008). Locally uniformly rotund norms in Banach spaces and their duals, Journal Functional Analysis, 254, 2023-2039.

[8] Haydon, R., Moltó, A. And Orihuela, J., (2007). Spaces of functions with countably many discontinuities, Israel Journal Math., 158, 19-39.

[9] Johnson, W. B. And Lindenstrauss, J., (2001). Basic concepts in the geometry of Banach spaces, Handbook of the geometry of Banach spaces, Vol. I, North-Holland, Amsterdam, 1-84.

[10] Kelley, J. L., (1975). General topology, Reprint of the 1955 edition [Van Nostrand, Toronto, Ont.], Graduate Texts in Mathematics, N. 27, Springer-Verlag, New York.

[11] Martínez Romero, J. F., (2007). Renormings in $C(K)$ spaces, Doctoral disertation, Valencia University.

[12] Martínez Romero, J. F., Moltó, A., Orihuela, J. And Troyanski S., (2007). On locally uniformly rotund renormings on $C(K)$ spaces. To appear in Canadian Journal Math.

[13] Moltó, A., Orihuela, J. And Troyanski, S., (1977). Locally uniformly rotund renorming and fragmentability, Proc. London Math. Soc., (3), 75, 619-640.

[14] Moltó, A., Orihuela, J., Troyanski, S. And Valdivia, M., (1999). On weakly locally uniformly rotund Banach spaces, J. Funct. Anal., 163, 2, 252-271.

[15] Moltó, A., Orihuela, J., Troyanski, S. And Valdivia, M., (2006). Continuity properties up to a countable partition, RACSAM, Rev. R. Acad. Cien. Serie A. Mat. , 100, (1-2), 279-294.

[16] Moltó, A., Orihuela, J., Troyanski, S. And Valdivia, M., (2009). A nonlinear transfer technique for renorming, Lecture Notes in Mathematics 1951, Springer Verlag, New York.

[17] Orihuela, J. And Troyanski, S., (2008). Devilles's Master Lemma and Stone discretness in renorming theory. To appear in Journal Convex Analysis.

[18] RAJA, M., (1999). On locally uniformly rotund norms, Mathematika, 46, 343-358.

[19] TroyansKi, S., (1971). On locally uniformly convex and differentiable norms in certain non separable Banach sapces, Studia Math., 37, 173-180.

[20] ZiZLER V., (2003). Non separable Banach spaces, Handbook of Banach spaces, Edt. Johnson and Lindenstrauss. Vol II, 1743-1816. North-Holland, Amsterdam.

\section{José Orihuela}

Departamento de Matemáticas. Facultad de Matemáticas.

Campus de Espinardo.

Universidad de Murcia.

E-30100 Espinardo. SPAIN.

joseori@um.es

\section{Stanimir Troyanski}

Departamento de Matemáticas. Facultad de Matemáticas.

Campus de Espinardo.

Universidad de Murcia.

E-30100 Espinardo. SPAIN.

stroya@um.es 